\title{
El CAMPO LABORAL DE LA ANTROPOLOGía
}

\section{en México}

\author{
Guadalupe Escamilla / Fernando I. Salmerón
}

Centro de Investigaciones y Estudios Superiores en Antropología Social (Ciesas)

\author{
LAURA R. VALLADARES \\ DEPARTAMENTO DE ANTROPOLOGÍA \\ Universidad AutónOma Metropolttana-IZTAPALAPa \\ fernando.salmeron@gmail.com
}

\begin{abstract}
Resumen
[N MÉXICO, LA ANTROPOlOGÍA TIENE UNA LARGA TRAYECTORIA DE DESEMPEÑO PROFESIONAL TANTO EEN El ÁM Bito ACADÉM ICO COM O FUERA DE ÉL. EN ESTE ARTíCULO P ONEM OS EL ACENTO EN EL TRABAJO DE AQUELOS P ROFESIONALES DE LA DISCIPLINA QUE SE DEDICAN A ACTIVIDADES DIFERENTES A LA INVESTIGACIÓN ACADÉM ICA. AB ORD AM OS EL TEM A A P ARTIR DE ASEVERACIONES DE ANTROP ÓLOGOS M EXICANOS QUE HAN DEFINIDO LA ACTIVIDAD P ROFESIONAL DE DIVERSA M ANERA, TANTO DESDE EL ÁM B ito ACADÉM ICO COM O DEL AP LCADO. PRESENTAM OS INFORM ACIÓN SOBRE LA DISTRIB UCIÓN DE LOS ANTROP ÓLOGOS ENTRE LOS DOS ÁM BITOS Y M ENCIONAM OS DIVERSOS TERRENOS DEL TRAB AJO ANTROP OLÓGICO EN MÉXICO EN LA ACTUALIDAD, SUB RAYANDO LA CRECIENTE DIVERSIDAD DE NUEVOS NICHOS Y LOS RETOS QUE ESTO REP RESENTA P ARA LOS JÓVENES ANTROP ÓLOGOS.
\end{abstract}

PALABRAS ClAVE: antropología aplicada, empleo de antropólogos, profesionalización, México.

\section{Professional anthropological work in Mexico}

\begin{abstract}
ANTHROPOLOGY IN MEXICO HAS A LONGSTANDING TRAJECTORY OF PROFESSIONAL INVOLVEMENT, BOTH IN Aacademia and public settings. In this essay we stress the work of those professionals of the discipline who work outside academic settings. We undertake the discussion starting from different perspectives expressed by Mexican anthropologists who have advanced contrasting views of anthropological work. We present information about current professional settings where anthropologists are employed in Mexico, stressing the growing diversity of these niches and the challenges that young anthropologists face.

KEY WorDs: Applied anthropology, employment for anthropologists, professionalization, Mexico.
\end{abstract}

\section{Revista Colombiana de Antropología}

Volumen 43, enero-diciembre 2007, pp. 387-4I8 
- N MÉXICO, LA ANTROPOLOGÍA TIENE UNA LARGA TRAYECTORIA DE DESEMPEÑO profesional tanto en el ámbito académico como fuera de él. Sus -antecedentes se remontan a la conquista, cuando la relación entre escribientes mesoamericanos y misioneros produjo una serie de relatos etnográficos de gran magnitud. Sin embargo, como disciplina, la antropología se consolidó a principios del siglo veinte, en relación con la formación del estado, la actividad intelectual y el diálogo con las corrientes intelectuales de Europa y Estados Unidos (véase Lameiras, I979; Arizpe, I993).

A cien años de que se dictara la primera cátedra de antropología en México, la disciplina ha transitado por diversos caminos; se han ampliado sus campos y sujetos de investigación, tanto por la complejización de las relaciones socioeconómicas, culturales y políticas, como por los ritmos y efectos producidos por la acelerada mundialización en las tres últimas décadas. Las aproximaciones teóricas han tenido cambios significativos también y, por supuesto, algunas continuidades. Hasta la década de I970, la antropología mexicana estuvo muy influenciada por las metropolitanas anglosajonas. A partir de entonces se inició una amplia discusión sobre la necesidad de descentrar los modelos teóricos y construir interpretaciones más acordes con nuestras realidades latinoamericanas y más cercanas a las necesidades y prioridades de los sujetos-actores de nuestras investigaciones. En este contexto se ha emprendido un diálogo todavía incipiente entre antropologías sur-sur y sur-norte desde nuevas visiones plurales ${ }^{1}$.

En este siglo de existencia, la praxis antropológica se ha modificado notablemente, pues los dos campos profesionales que se configuraron nítidamente a lo largo de esta centuria -uno dedicado a la formación e investigación en universidades y centros

I. Sobre las iniciativas de comunicación entre las antropologías mundiales se destaca la reciente creación del Consejo Mundial de Asociaciones de Antropología (WCAA), en el que han tenido un papel destacado antropólogos del sur como el brasilero Gustavo Lins Ribeiro. Sobre los miembros y actividades de la red se puede consultar su sitio web http://www.wcaanet.org/ de investigación especializados en antropología, y otro vinculado al trabajo en instituciones públicas dedicadas a la política indigenista, cultural y patrimonial del país- se han diversificado, especialmente durante las dos últimas décadas. Esto se debe, en primer lugar, a que los espacios institucionales para la investigación y la docencia no han crecido al ritmo que los nuevos profesionales demandan. Por otra parte, de forma paralela se han abierto nuevos nichos laborales en otras instituciones públicas, 
organismos paraestatales y no gubernamentales. Así mismo, la apertura teórico-práctica en aspectos importantes y novedosos como la promoción y la difusión de los derechos humanos o la procura de justicia, entre otros, han configurado la posibilidad de incidir en el mercado laboral con miradas novedosas desde la antropología social contemporánea. Estos cambios, por su parte, suponen también diferentes retos a los que haremos alusión en este ensayo.

En este texto ${ }^{2}$ nos proponemos presentar una reflexión sobre el desarrollo reciente del campo laboral de la antropología mexicana. Nos situamos en un momento histórico en el que la práctica de la antropología ha salido de los que eran sus espacios "naturales" en México: la política indigenista del estado y los centros de investigación académica. Para entender estos cambios presentamos un breve panorama sobre los inicios de la enseñanza de la antropología en México y su relación con el estado, resumimos dos de los planteamientos centrales que dieron lugar 2. Este artículo fue preparado originalmente para ser presentado en el panel de colegios profesionales titulado "Prácticas profesionales, desarrollos laborales", en el marco del XI Congreso nacional de antropología de Colombia, celebrado en Santa Fe de Antioquia, entre el 23 y el 25 de agosto de 2005. Posteriormente fue revisado para su publicación. Agradecemos los comentarios de dos dictaminadores anónimos que permitieron mejorar el texto.

Los autores encabezan la mesa directiva del Colegio de Etnólogos y Antropólogos Sociales, A. C. (Ceas).

a esta diversificación y presentamos las características generales de los lugares donde hoy se forman los antropólogos. Con estos antecedentes, exponemos cómo se han conformado los principales espacios de desarrollo profesional durante las últimas décadas y hacemos una breve reflexión desde la perspectiva del colegio profesional que tenemos la responsabilidad de impulsar, el Ceas. Las conclusiones recuperan de forma analítica cuáles han sido los principales cambios reseñados, cómo vemos el futuro laboral de la antropología profesional en México y de qué manera un colegio profesional como el Ceas puede incidir en esta problemática.

\section{LA INSTITUCIONALIZACIÓN}

\section{DE lA ANTROPOlOGía EN MÉxico}

N MÉXICO, LA IMPARTICIÓN DE CLASES ESPECIALES DE HISTORIA, ARQUEO-
logía y etnología tuvo inicio en I9O6 en el Museo Nacional,
gracias a la iniciativa de Nicolás León. Posteriormente, con la 
fundación de la Escuela Internacional de Arqueología y Etnología Americanas (I9II) ${ }^{3}$ se trasladaron algunas de las cátedras del Museo Nacional a la Escuela de Altos Estudios en 1915. Estos esfuerzos pueden situarse como los antecedentes de la formación de antropólogos en el país ${ }^{4}$.

Sin embargo, no fue sino en 1938, al crearse el Departamento de antropología dentro de la Escuela Nacional de Ciencias Biológicas

3. La Escuela Internacional fue impulsada por Franz Boas.

4. Véase Mayer Guala, Claudio. "La Escuela Internacional de Arqueología y Etnología Americanas y los orígenes de la antropología mexicana a principios del siglo $x x^{\prime \prime}$. Enah, tesis profesional, 1976 .

del Instituto Politécnico Nacional (IPN), cuando se formalizó un programa específico para formar profesionales en esta disciplina con una meta y unos perfiles definidos socialmente. En I942, la tarea de formar antropólogos se trasladó al recién creado Instituto Nacional de Antropología e Historia (Inah), y fue en este marco que se fundó la Escuela Nacional de Antropología e Historia (Enah), que ha sido, sin duda, la institución que más importancia e impacto ha tenido en la formación de antropólogos en México. La Escuela adquirió de parte de la Secretaría de Educación Pública (SEP) su carácter nacional y el monopolio de la formación de antropólogos en el país, que perdió en 1957 cuando se creó la carrera en la Universidad Veracruzana. Posteriormente se han creado muchos otros programas en las universidades autónomas de Yucatán, estado de México, Morelos y Metropolitana en el Distrito Federal (DF), por citar algunos, particularmente a partir de las décadas de I970-I980, pero el diseño de los planes de estudio estuvo muy influenciado por lo que acontecía en la Enah.

\section{LA FORMACIÓN DE ANTROPÓLOGOS EN MÉXICO CONTEMPORÁNEO Y EL MERCADO DE TRABAJO}

L INICIO DEL Siglo VEINTIUNO EXISTEN EN MÉxico veINTICUATRO ESCUELAS
o facultades en las que se forman antropólogos, veintiuna de
origen público y tres privadas. Prácticamente en todo el terri-
torio nacional es posible estudiar alguna de las especialidades de
la antropología: en la zona centro-norte del país están la Enah-
Chihuahua; la Universidad Autónoma de Zacatecas (Unidad de 
antropología); la Universidad de Colima (solamente licenciatura en lingüística); la Universidad Autónoma de Sonora (solamente licenciatura en lingüística); el Centro Educativo Universitario Panamericano en Monterrey, Nuevo León (solamente licenciatura en antropología); Coordinación de Ciencias Sociales y Humanidades de la Universidad Autónoma de San Luis Potosí; Programa de Estudios Antropológicos del Colegio de San Luis Potosí (Colsan); Centro de Investigación en Ciencias Sociales de la Universidad de Guanajuato y Centro de Estudios Antropológicos del Colegio de Michoacán (Colmich).

En la región central existen nueve instituciones, de las cuales cinco tienen su sede en el DF: la Escuela Nacional de Antropología e Historia (Enah); el Departamento de antropología de la Universidad Autónoma Metropolitana Iztapalapa (UAM-I); el Centro de Investigaciones y Estudios Superiores en Antropología Social (Ciesas); el posgrado en antropología de la Facultad de filosofía y letras, en el que está inscrito el Instituto de Investigaciones Antropológicas de la Unam (IIA-Unam); y la Universidad Iberoamericana. Cuatro más se ubican en los estados de México (Facultad de antropología de la Universidad Autónoma del Estado de México); en Querétaro (Facultad de filosofía de la Universidad Autónoma de Querétaro); en Puebla (Colegio de antropología social de la Benemérita Universidad Autónoma de Puebla); y en Morelos (Departamento de antropología de la Facultad de humanidades de la Universidad Autónoma del Estado de Morelos).

En los estados sureños existen posibilidades de estudiar antropología en Guerrero (Programa de estudios antropológicos de la Universidad Autónoma de Guerrero); en Veracruz (Facultad de antropología de la Universidad Veracruzana); en Chiapas (Área de antropología de la Facultad de ciencias sociales de la Universidad Autónoma de Chiapas) y (División de procesos sociales de la Universidad Intercultural de Chiapas); en Yucatán (Facultad de ciencias antropológicas de la Universidad Autónoma de Yucatán); y en Quintana Roo (Departamento de ciencias sociales de la Universidad de Quintana Roo).

En el conjunto, la oferta abarca desde el nivel de licenciatura hasta el de doctorado, y comprende alguna de las siete especialidades reconocidas: antropología social, etnología, etnohistoria, antropología física, arqueología, lingüística e historia. También han empezado a surgir especialidades como comunicación intercultural, promoción cultural, lengua y cultura o lingüística 
aplicada. De acuerdo con datos de la Asociación Nacional de Universidades e Instituciones de Educación Superior (Anuies), en 2003 había 5.027 estudiantes inscritos en las diferentes especialidades en el país: 38,3\% hombres y 6I,7 mujeres. En ese mismo año egresaron 456 nuevos profesionales, de los cuales un poco más de 45\% eran antropólogos sociales.

La mayor parte de estas escuelas se encuentran agrupadas en la Red Mexicana de Instituciones de Formación de Antropólogos (Red MIFA), creada en 2000 con el objeto de generar la cooperación interinstitucional, fortalecer, profundizar y agilizar el intercambio académico entre las instituciones. Los propósitos específicos incluyen favorecer la realización y el desarrollo de investigación científica en el campo antropológico; impulsar procedimientos de movilidad docente y estudiantil; establecer y gestionar mecanismos de colaboración que permitan coadyuvar a la preparación de estudiantes y la convalidación de carácter regional, nacional e internacional de sus estudios. Se pretende con ello lograr el establecimiento de un sistema de reconocimiento de créditos y líneas curriculares entre las instituciones participantes. Los integrantes de la red se comprometen así a brindar la ayuda necesaria por medio de asesorías, apoyos técnicos e intercambio de servicios en las diversas áreas con las que cuentan las instituciones. Por último, asumen la tarea de desarrollar actividades conjuntas entre las escuelas, las facultades, los institutos, los departamentos y los programas que imparten antropología en materia de docencia, investigación y difusión en las áreas de su competencia y programas específicos de su interés. Se trata, en síntesis, de una red importante que permite estrechar lazos entre antropologías regionales y discutir y proponer soluciones para problemas compartidos ${ }^{5}$.

A pesar de estos esfuerzos encomiables, el número exacto de profesionales de la antropología existentes en México no puede determinarse con exactitud, lo que constituye un reto pendiente

5. Para mayor información sobre esta red pueden consultarse las direcciones de internet: http:// www.vaemex.mx/pwww/ant/ y http://vam-antropologia.info/web para los colegios y asociaciones profesionales. Por ejemplo, al cumplirse el 60 aniversario de la Enah, en 1998, se estimaba que había graduado alrededor de mil doscientos antropólogos en las siete especialidades de nivel licenciatura y en los cuatro posgrados que entonces existían, pero no se conocía el número total de alumnos que se habían inscrito 
en sus aulas ni los que habían egresado en todo el periodo. Más difícil, y no por ello menos urgente, es conocer el número exacto de los profesionales de estas disciplinas que se han insertado en el mundo laboral y sus condiciones de trabajo. Las estadísticas compiladas por la Secretaría del Trabajo y Previsión Social y el Observatorio Laboral ${ }^{6}$, que toman como referencia la Encuesta nacional de ocupación y empleo trimestral, STPS-Inegi, agrupan al conjunto de disciplinas antropo-

6. Véase http://www.observatoriolaboral.gob. $\mathrm{mx} / \mathrm{p}$ PanoramaCar.asp? $\mathrm{clave}=253$ lógicas bajo el rubro: "Antropología y arqueología y etnología”, e indican que entre 1997 y 2006 egresaron de las instituciones superiores un promedio de quinientas personas por ciclo lectivo, en su mayoría mujeres, lo que da un total de cuatro mil quinientas en nueve años, aproximadamente. Sin embargo, la cifra de alumnos inscritos en esos mismos años en el conjunto de instituciones y para el mismo bloque de carreras va de cuatro mil a cuatro mil cuatrocientas por año escolar.

Estas mismas fuentes indican que los que estaban ocupados en áreas afines a los estudios que realizaron -clasificados bajo antropología, arqueología y etnología-iban de seis mil ochocientos en 1999 a cinco mil quinientos al primer trimestre de 2007 , con máximos de once mil cuatrocientos y trece mil trescientos en 200I y 2002, respectivamente. La ocupación fundamental al primer trimestre de 2007 de este sector de población fue clasificada como: profesionales en ciencias sociales (2I,I\%); profesores universitarios y de otros establecimientos de enseñanza superior (I6,6\%); profesores de preparatorias y escuelas de nivel medio superior $(\mathrm{I} 6,4 \%)$; y jefes de departamento, coordinadores y supervisores (4,6\%), para un subtotal de 60,7\%. El restante 39,3\% declaró que estaba empleado en actividades no relacionadas con los estudios que realizó.

En lo que se refiere a su distribución regional en el país, 48,7\% se ubicaba en los estados del centro de la República: Distrito Federal, Hidalgo, México, Morelos, Puebla y Tlaxcala; 38,6\% en los del sur-sureste: Campeche, Chiapas, Guerrero, Oaxaca, Quintana Roo, Tabasco, Veracruz y Yucatán; I2,3\% en los del centro-occidente: Aguascalientes, Colima, Guanajuato, Jalisco, Michoacán, Nayarit, Querétaro, San Luis Potosí y Zacatecas; mientras que el o,4\% restante trabajaba en los estados de noreste y del noroeste de México.

Con base en estos datos, podemos afirmar que la oferta de trabajo para los egresados de las disciplinas antropológicas se ha 
modificado sustancialmente en las últimas décadas respecto a lo que se esperaba cuando se instituyó la carrera. Desde su establecimiento como una disciplina institucionalizada, la antropología en México se consideró una profesión destinada a la orientación y ejecución de la acción pública y del cuidado y resguardo del patrimonio cultural y arqueológico. Como afirma Virginia Molina (s. f.), la antropología social era una ciencia que proporcionaba a sus practicantes herramientas analíticas para desempeñarse como agentes o consultores de las agencias gubernamentales encargadas del cambio social planificado. De hecho, no se hacía distinción en el currículo ni en la orientación entre antropología social y antropología aplicada. Cuando en I95I, en coordinación con el Instituto Nacional Indigenista (INI), se estableció la especialidad en antropología aplicada, se incluyeron cursos que permitían desarrollar las herramientas para esta aplicación. De acuerdo con esta autora, los profesores combinaban sus actividades de investigación en el Inah con la práctica de la antropología en apoyo de la política indigenista, la vivienda, la nutrición o el desarrollo de la comunidad. De esta forma, muchos de los estudiantes de antropología se formaron en esta práctica del trabajo antropológico. Por otra parte, la información nos muestra que a lo largo de los últimos treinta años el empleo académico creció y se expandió en el interior de país, tanto en universidades como en institutos y centros de investigación, ampliando así la oferta original que ofrecían el Instituto Nacional Indigenista, el Instituto de Antropología e Historia y la Enah.

Además de ello, en la actualidad los egresados de las carreras de antropología han sido convocados para distintos proyectos que van desde la investigación de campo en áreas remotas y con presencia indígena, hasta investigaciones de opinión, evaluación o ampliación de mercado. En otras ocasiones han sido los propios antropólogos quienes proponen sus conocimientos y experiencia a asociaciones civiles y organismos no gubernamentales para emprender proyectos y programas en los que la visión y los conocimientos de la disciplina son importantes. Lo que sin duda continúa siendo una constante en el quehacer profesional de la antropología es su metodología, es decir, su visión comparativa y el trabajo cercano con los actores sociales que estudia, así como su compromiso con la equidad social y la democracia. 


\author{
Gonzalo Aguirre Beltrán, \\ Ángel Palerm \\ Y LA ANTROPOLOGÍA APLICADA
}

V

OLVAMOS AHORA A LAS DIFERENTES PRÁCTICAS PROFESIONALES QUE HA tenido la antropología en México, desde su vertiente indigenista hasta las más recientes denominadas como "antropología comprometida” y “antropología dialógica-crítica colaborativa”. Todas ellas sostienen la importancia de la aplicación de los conocimientos en beneficio de los propios grupos con los que se trabaja. Sin embargo, históricamente han existido diferencias fundamentales relacionadas con la mejor manera de llevar a efecto estos propósitos, y muchas de ellas derivan del cómo concebir la distinción entre teoría y práctica en el quehacer antropológico. Analizarlas detalladamente en las publicaciones de los antropólogos mexicanos requeriría un trabajo que no podemos hacer en este texto. Por tanto, trataremos de resumir de manera muy breve tres corrientes significativas en este tema, que hoy orientan la mayor parte del trabajo profesional que se lleva a cabo.

El “antropólogo aplicado” más conocido de México es Gonzalo Aguirre Beltrán, quien, a pesar de haber contribuido con una larga lista de publicaciones a la discusión académica, trabajó muy pocos años en centros de investigación y formación académica. La mayor parte de su producción la desarrolló mientras trabajaba para el gobierno mexicano: en la Secretaría de Gobernación, el Instituto Nacional Indigenista, el Instituto Indigenista Interamericano y la Secretaría de Educación Pública.

Aguirre Beltrán consideraba que "no debe hacerse una distinción entre antropología aplicada y antropología no aplicada”. Advertía en esta distinción "una idea aristocrática de división de las disciplinas" y siempre sostuvo que "la teoría emerge de la práctica. Y mientras no haya práctica -y la práctica quiere decir eso; ciencia aplicada- no puede haber, propiamente, teoría”. Afirmaba, entonces, que la escuela de antropología mexicana, desde Manuel Gamio, estableció que se hace práctica y, a partir de ella, se extraen normas que sirven para guiar la investigación y la aplicación. De acuerdo con esta idea sostenía que "toda la teoría indigenista salió de la práctica; no es más que práctica llevada a nivel científico". En este sentido, la escuela mexicana se distanciaba de las ideas de Boas, quien estaba declaradamente 
en contra de la aplicación de la antropología en los países coloniales. Esto implicaba que la disciplina preparaba profesionales para la práctica y para la reflexión crítica sobre esa práctica, aun cuando no necesariamente fueran las mismas personas las que desempeñaran esas actividades de manera simultánea (Salmerón, entrevista a Aguirre Beltrán, I990: 22I-224).

Ángel Palerm, considerado por Aguirre Beltrán como uno de sus interlocutores más destacados, defendió siempre la importancia de la praxis antropológica como un elemento central de la disciplina. Sin embargo, establecía una distinción entre la antropología como ciencia y el trabajo de promoción del desarrollo. Palerm defendía el compromiso del antropólogo con la justicia social y la promoción del cambio estructural, y justamente veía como problemático el papel del antropólogo como funcionario o promotor, porque esto implicaba un compromiso con un proyecto particular que dificultaba la necesaria tarea de hacer un examen crítico del problema global.

Palerm (1993) escribió que "la antropología no es, no ha sido nunca, ni podrá llegar a ser, una ciencia pura, lo mismo en el sentido literal que en el metafórico de la palabra 'pura”. En el sentido corriente del término no podría ser ciencia pura,

sencillamente porque desde su nacimiento (...) la antropología se ha caracterizado por una decidida vocación para aplicar y usar de alguna manera sus conocimientos. Es más, podría uno llegar a decir que el conocimiento antropológico, y con él la antropología, se generaron, precisamente ante ciertas necesidades de naturaleza muy peculiar del comercio, de la guerra, del proselitismo religioso y de la conquista.

Esta asociación estrecha de la antropología con los "esquemas de dominio y conquista" ha llenado a la disciplina de "impurezas, en el sentido no tan metafórico de la palabra”. Sin embargo, advierte Palerm,

no toda la aplicación de la antropología, ni tampoco toda actividad antropológica, ha tenido o tiene esa desagradable naturaleza. (...) Existe a la vez otra historia más honorable y digna. (...) Es la historia del envolvimiento de la antropología con las aspiraciones de una sociedad más libre y justa, con la lucha contra el racismo y la explotación humana en cualquier forma (Palerm, 1993: 36I-362). 
Actualmente, una perspectiva más reciente que tiene una influencia muy importante de la antropología posmoderna sostiene que el investigador no puede lograr la objetividad plena. De acuerdo con esta perspectiva, la verdad no es algo que uno pueda colectar en la zona de estudio, como haría un biólogo con un ejemplar de flora o fauna. Postula que, en el caso de los procesos sociales, tratamos más bien con una historia, una construcción social, que se escribe a partir de la realidad observada y percibida por quienes llevan a cabo el estudio. Las percepciones de los sujetos con los que trabajan los antropólogos suelen ser diferentes y es más preciso un análisis que logra incorporar las distintas miradas. Desde esta perspectiva, la diversidad no sólo es un dato empírico, sino también una fuente de riqueza en la construcción de conocimiento y en el diseño de programas de desarrollo. En este sentido, la disciplina promueve una posición que estimula a quienes trabajan en el desarrollo a escuchar las historias de otras personas, a prestar atención a puntos de vista divergentes y a nuevas maneras de ver y hacer las cosas (Gardner y Lewis, I996). Incluso puede llevarse esta posición un poco más lejos hasta involucrar a los sujetos en el proceso de investigación. La propuesta de una investigación colaborativa, como la que defiende Aída Hernández (Hernández Castillo, 2003), implica reconocer la parcialidad de la perspectiva del investigador, "la multiplicidad de posiciones de los sujetos que marcan las identidades de los actores sociales y sus relaciones de subordinación y las limitaciones de nuestros conocimientos situados”. Una antropología dialógica-crítica que:

no se propone transformar la realidad en base a (sic) un método o teoría que se considere infalible, sino reflexionar y deconstruir junto con los actores sociales con quienes trabajamos problemáticas de una realidad social compartida. A partir de estos diálogos elaborar conjuntamente una agenda de investigación que haga nuestro conocimiento relevante para los actores sociales con quienes trabajamos (Hernández Castillo, 2003).

Como podemos apreciar, existe una reflexión sobre la interpretación teórica entre un grupo de antropólogos que defienden desde la trinchera académica un discurso epistémico en el que la praxis, si bien distinta a la tradicional institucional o indigenista, enfatiza el trabajo cercano con los actores sociales con los que 
compartimos problemáticas, intereses y vocaciones. Esta es, sin duda, una reflexión que es compartida también, aun cuando sin diálogo entre ellos, por los antropólogos indigenistas y los que se insertan en diferentes campos laborales. De aquí surgen diversos interrogantes como los siguientes: ¿cómo está constituido el ámbito institucional en el que se forman los nuevos antropólogos? ¿Se discuten las distintas formas y prácticas que nuestro quehacer profesional implican? ¿Se requieren nuevos contenidos curriculares para formar antropólogos con conocimientos y habilidades que los preparen para los requerimientos de los nuevos campos profesionales? No pretendemos dar respuesta a estos interrogantes básicos. Sin embargo, con miras a apuntar algunos datos que nos acerquen a estos cuestionamientos, en los siguientes apartados hablaremos sobre nuevos campos profesionales abiertos a la práctica de la antropología en México.

\section{Espacios actuales \\ DE DESARROLLO PROFESIONAL \\ DE LOS ANTROPÓlogos EN MÉxico}

${ }^{\mathrm{N}}$

N ESTE CONTEXTO DE COMPROMISO DE LOS ANTROPÓLOGOS CON LOS GRANdes problemas y desafíos del México contemporáneo, durante las últimas décadas las nuevas generaciones han incursionado en novedosos campos profesionales. Sin duda esto responde en buena medida a la relativa contracción de la oferta tradicional de empleo por parte del estado y sus agencias, la principal fuente durante la primera mitad del siglo veinte. Así mismo, es necesario reconocer que cada día es más difícil que los egresados de nuestra disciplina puedan incorporarse a las labores académicas y de investigación, debido a dos circunstancias de compleja resolución. Por una parte, las plazas de nueva creación en las instituciones que dedican sus esfuerzos a estos campos no han crecido al ritmo de la disponibilidad de profesionales formados. Por otra, las dificultades para garantizar un retiro digno a los pro-

7. Véase al respecto Bensusán y Ahumada Lobo (2006).

fesores en edad de jubilación han impedido la liberación de estas plazas en los centros de enseñanza e investigación ${ }^{7}$. Por tanto, ha sido necesario incorporarse en áreas poco exploradas en términos profesionales. Estos nuevos 
espacios están relacionados generalmente con la aplicación de los conocimientos y las metodologías antropológicas en instituciones públicas, organizaciones no gubernamentales, organizaciones sociales, consultorías y fundaciones, entre otras. Esta inserción ha enriquecido ampliamente la labor de dichos organismos con la visión, la experiencia y el compromiso de los profesionales de las ciencias antropológicas.

Sin embargo, es necesario reconocer también que en México existe todavía una suerte de desprecio por aquello que se considera como "praxis" pura, es decir, por la labor de aquellos colegas que están ejerciendo su profesión en campos diferentes a la reflexión, la interpretación y el análisis teórico (véase la discusión en Báez, 2005). En este sentido la experiencia de grandes antropólogos en diversos campos ha sido la que ha hecho visibles algunos nuevos campos profesionales. A partir de esta experiencia los profesionales del mundo académico han sido cuestionados acerca del tipo de formación que están imponiendo a los jóvenes. Colegas como Virginia Molina (s. f.) han insistido enfáticamente en la necesidad de formar a los nuevos antropólogos en temas, métodos y análisis que los preparen para acceder con bases sólidas a los nuevos retos profesionales que la sociedad y el mercado exigen de la antropología. En este marco, cobra importancia la presencia aún escasa de los colegios de profesionales como una forma de normalizar estos requerimientos de actualización de temáticas, adquisición de habilidades y dominio de nuevas herramientas metodológicas. Una primera tarea en este sentido es la de acercar a los antropólogos del país que se desarrollan en esferas distintas en su ejercicio profesional y que tienen poco o ningún diálogo entre sí.

A pesar de esta dispersión relativa, es evidente la incorporación de antropólogos en diversas áreas de actividad profesional. A continuación examinaremos la forma en la que se ha dado esta participación en nuevos nichos laborales y las que vemos como las principales razones para ello. De entrada, sin embargo, podemos advertir dos grandes elementos novedosos que inciden directamente en el papel que los antropólogos pueden desempeñar. Por una parte, podríamos decir que se ha instaurado una suerte de incipiente "política de la identidad" que se ha traducido en modificaciones constitucionales que reconocen derechos a los pueblos indígenas. Por otra, hemos asistido a los primeros pasos en una suerte de política afirmativa que ha intentado, aun cuando con poco éxito, que todas las dependencias gubernamen- 
tales impulsen proyectos de atención a los grupos vulnerables -entre los que se incluyen los pueblos indígenas-, reduzcan las inequidades de género, hagan conciencia de la diversidad y eviten la discriminación. Como resultado se han abierto oportunidades de intervención de los antropólogos en estas áreas, tanto en los espacios de la administración gubernamental en sus diferentes niveles, como en las organizaciones sociales, civiles e incluso privadas, como veremos a continuación.

\section{LAS INSTITUCIONES COMO NICHO DE TRABAJO PARA ANTROPÓLOGOS}

OMO ES CONOCIDO, Y COMPARTIDO POR AMÉRICA LATINA, DESDE LA DÉCADA
de I99O el gobierno mexicano ha hecho suyo el discurso del
respeto a la pluralidad política y sociocultural. En concordancia se han dado algunos avances en la construcción de un modelo multicultural-liberal, que se ha traducido en la institucionalización del llamado multiculturalismo, mediante la introducción de cambios en la política indigenista o la creación de espacios institucionales para hacer operativa una incipiente política de la identidad (Dietz, 2003; Gros, 2000).

Hoy en día la discusión sobre las políticas públicas para los indígenas ha dado un nuevo giro a partir de los gobiernos de corte neoliberal que han orientado la política de desarrollo social hacia una estrategia focalizada en la atención de la población en condición de pobreza y de pobreza extrema, como característica fundamental para la distribución de los apoyos económicos gubernamentales destinados a reducir la inequidad y marginación, mediante programas como el denominado "Oportunidades" o los fondos regionales que maneja la Comisión Nacional para el

8. Creada en 2003, la CDI sustituyó al Instituto Nacional Indigenista en el marco de la reforma del estado promovida por el presidente panista Vicente Fox Quezada (2000-2006).

9. Sobre los retos de la antropología aplicada véase la revista Cuicuilco, nueva época, I2 (35), septiembre-diciembre 2005 .

institucional se mantione, aun cuando buscando cada vez más un encuentro que aligere las distinciones entre ambas ${ }^{9}$. 
A partir del levantamiento del Ejército Zapatista de Liberación Nacional (EZLN) en I994, el reconocimiento de algunos derechos de los pueblos indígenas ha inducido cambios legislativos significativos aun cuando limitados ${ }^{10}$. En la actualidad, dieciocho de los treinta y un estados de la República han reconocido en sus respectivas constituciones la composición pluricultural y multilingüe del pueblo mexicano y han legislado en correspondencia con la nueva ley indígena, que reconoce el derecho de los indígenas a la autonomía (López Bárcenas, 2002; Valladares, 2003). Sin embargo, sólo en cinco de esos estados se ha elaborado una ley reglamentaria que puntualiza derechos, competencias y sujetos de derecho. En este proceso de reformas legislativas varios antropólogos han participado como asesores, ya sea del Congreso de la Unión o de los Congresos locales. En otras ocasiones, diversos especialistas en derechos indígenas han aportado su experiencia en foros, seminarios, talleres, encuentros y consultas, tanto las convocadas por el aparato legislativo de la nación, como por organizaciones indígenas y organizaciones no gubernamentales.

La apertura de estos espacios de participación profesional no ha eliminado los que habían ocupado los antropólogos en el diseño, la construcción y la ejecución de políticas públicas dirigidas a la población indígena, aun cuando ahora se comparten con especialistas de otras disciplinas. El principal empleador en esta materia es la Comisión Nacional para el Desarrollo de los Pueblos Indígenas (CDI), que resultó de la conversión y reestructuración del antiguo Instituto Nacional Indigenista al inicio del decenio 2000. Esta institución del gobierno federal tiene una amplia variedad de programas de atención a la población indígena y de promoción de la diversidad cultural. Entre ellas se encuentra

IO. El gran cambio legislativo en este rubro fue la modificación a la constitución federal mexicana en materia indígena, realizada en 200I. Esta modificación reconoce algunos derechos indígenas fundamentales, pero ha sido seriamente cuestionada por amplios sectores sociales y, sobre todo, por las organizaciones políticas indígenas, que la consideraron acotada y alejada de los reclamos autonómicos de esos pueblos.

II. Este programa se inició el Io de marzo de I979, cuando el Instituto Nacional Indigenista puso en el aire las transmisiones de la radioemisora XEZV "La Voz de la Montaña", en Tlapa de Comonfort, Guerrero. Hoy, veinticinco años después, el Sistema de Radiodifusoras Culturales Indigenistas (SRCI) comprende veinte emisoras que transmiten en la banda de amplitud modulada y cuatro más en la banda de frecuencia modulada. En estas radiodifusoras se transmite en treinta y una lenguas indígenas. el Sistema de Radiodifusoras Culturales Indigenistas (SRCI) ${ }^{11}$, que durante veinticinco años han transmitido y difundido la lengua, la cultura, las tradiciones y la música de los pueblos indígenas. 
Otra fuente importante de empleo para los antropólogos en México ha sido la Secretaría de Educación Pública, particularmente en las áreas que atienden a la población rural e indígena, como son la Dirección General de Educación Indígena y el Consejo Nacional para el Fomento Educativo (Conafe), aun cuando hay otros espacios en la Coordinación General de Educación Intercultural y Bilingüe (CGEIB) y los diversos programas de atención a indígenas, migrantes y población rural dispersa. La CGEIB ha impulsado en los últimos años nueve universidades interculturales y programas de bachillerato intercultural en diferentes estados del país ${ }^{12}$. En este mismo campo de la educa-

I2. Las universidades son las siguientes: Universidad Autónoma Indígena de México, Mochicahui, Sinaloa; Universidad Intercultural de Chiapas, San Cristóbal de las Casas; Universidad Intercultural del Estado de México, San Felipe del Progreso; Universidad Intercultural Indígena de Michoacán, Pátzcuaro; Universidad Intercultural de la Montaña de Guerrero, Tlapa de Comonfort; Universidad Intercultural de Puebla, Huehuetla; Universidad Intercultural de Tabasco, Oxolotán; Universidad Veracruzana Intercultural -cuatro planteles en el estado-; y Universidad Intercultural Maya, en José María Morelos, Quintana Roo. Los bachilleratos están distribuidos en diversas entidades del país. Véanse los informes presentados en el Encuentro regional sobre educación superior de los pueblos indígenas en América latina, realizado en la ciudad de México el 27 de septiembre de 2003. Se puede consultar también el trabajo de Rebolledo (200I). Para tener una visión sobre los ejes rectores de la educación indígena en México véase Schmelkes (2003), SEP-CGEIB (2006) y SEP-CGEIB (2004). ción, el Io de noviembre de 2003 entró en vigor la ley general de derechos lingüísticos de los pueblos indígenas, que reconoce como lenguas nacionales tanto a las lenguas indígenas como al español, pues a la letra señala que "Las lenguas indígenas que se reconozcan en los términos de la presente ley y el español son lenguas nacionales por su origen histórico, y tienen la misma validez en su territorio, localización y contexto en que se hablen". En la elaboración de esta nueva ley fue fundamental la presencia de especialistas de nuestra disciplina.

Otro campo de trabajo, estrechamente ligado al anterior, se encuentra en el Consejo Nacional para la Cultura y las Artes, institución que coordina museos, estrategias de difusión de la diversidad cultural mexicana y el fomento de las tradiciones y la cultura popular. Entre los lugares de empleo se encuentran la Dirección General de Culturas Populares y el Museo de Culturas Populares, que tienen a su cargo el fomento de las tradiciones y la cultura popular, y, por supuesto, el Instituto Nacional de Antropología e Historia. En este último hay tanto funcionarios como investigadores en todas las ramas de la disciplina, centrados fundamentalmente en tareas ligadas con el estudio y la conservación del patrimonio prehispánico, histórico y cultural, así 
como en tareas de museografía para salvaguardar y difundir este patrimonio nacional. De acuerdo con información dada a conocer por la directora de la Coordinación Nacional de Antropología, Gloria Mercader, esta institución cuenta con más de doscientos investigadores distribuidos en todo el país, que tienen registrados doscientos cincuenta diferentes proyectos de investigación. A esta cifra se deben sumar los profesores-investigadores de la Enah, los de la fototeca del Inah y todos aquellos investigadores contratados para algún proyecto en específico ${ }^{13}$.

En épocas recientes algunos antropólogos físicos se han acercado a la medicina forense y molecular, colaborando con las agencias de procura de justicia. Otros profesionales se han involucrado en las áreas de salud, particularmente en los programas de atención al medio rural e indígena. La Secretaría de Salud ha invitado a antropólogos a asesorar sobre la estrategia adecuada para hacer campañas de salud, de vacunación y de control de la natalidad considerando las culturas y las tradiciones de las poblaciones indígenas. Una mención aparte merecen los programas dirigidos a impulsar modelos médicos en donde los conocimientos étnicos de parteras y curanderos puedan convivir con los programas de la medicina alópata. Debemos subrayar la importante participación de antropólogos de la medicina en el desarrollo de programas de atención médica en la situación intercultural ${ }^{14}$, especialmente porque han llevado al gobierno federal a plantear la necesidad de incorporar un enfoque inter-

I3. Véase el reporte completo en "Sala de prensa del Consejo Nacional para la Cultura y las Artes" (CNCYA), 30 de abril de 200I.

I4. Véase Freyermuth (s. f.) y Lerín (s. f.). cultural en la formación del personal directivo y operativo de la Secretaría de Salud y de instituciones afines.

Otro empleador en el gobierno federal es la Secretaría de Desarrollo Social (Sedesol), particularmente en las áreas de políticas públicas que promueven la igualdad de oportunidades para el desarrollo social y humano, el Fondo Nacional para el Fomento de las Artesanías (Fonart), el Instituto Nacional de Desarrollo Social (Indesol) y el Programa de Desarrollo Humano "Oportunidades”. En la Secretaría de la Reforma Agraria han participado también antropólogos en diversos niveles de la estructura, desde cargos de dirección hasta la coordinación de los archivos históricos que resguardan la memoria documental sobre la tenencia de la tierra en México. Hoy tenemos también antropólogos que aplican sus conocimientos en otras secretarías de estado tales como la 
Secretaría del Medio Ambiente (Semarnat), en donde se creó un Programa especial de atención a indígenas 2002-2006, que involucra programas para la preservación y explotación sustentable de los territorios indígenas en manos de los propios indígenas.

El empleo de antropólogos en las agencias del gobierno federal tiene una contraparte importante en las instituciones de los gobiernos de los estados de la Federación. Aun cuando estas administraciones son considerablemente más reducidas y en ellas los antropólogos han tendido a concentrarse en las actividades de los institutos de cultura y en las secretarías de educación, hay cada vez más ejemplos de otras actividades. Recientemente un miembro del gremio se convirtió en gobernador de uno de los estados de la Unión y hemos contado con varios miembros de los cuerpos legislativos, tanto federales como locales.

En algunas de las empresas paraestatales mexicanas han participado también los antropólogos, sobre todo en los grandes proyectos de explotación petrolera o producción de energía eléctrica. Es de destacarse la presencia de antropólogos en la Comisión Federal de Electricidad (CFE), quienes elaboran, entre otras cosas, diagnósticos del impacto social y ambiental provocado por la construcción de proyectos hidroeléctricos y proponen estrategias para reducir y compensar los efectos de dichas obras. Si bien hasta ahora se trata de experiencias limitadas y de un todavía reducido número de antropólogos en estas instituciones, son muestra de una mayor sensibilidad gubernamental a la diversidad cultural de la nación mexicana.

Por otra parte, debemos reconocer que estos cambios en las políticas públicas y en la legislación nacional han obedecido, en muchos casos, a los compromisos gubernamentales establecidos con instituciones internacionales. Organismos multilaterales como la Organización de las Naciones Unidas, la Organización Internacional del Trabajo, la Unesco, el Banco Interamericano de Desarrollo y particularmente el Banco Mundial han sido muy insistentes en la necesidad de atender puntualmente las demandas sociales de la población en general y de los grupos indígenas en particular. Estos acuerdos y la presión popular de los movimientos sociales han logrado la implantación de acciones concretas para salvaguardar los derechos de las minorías y para mejorar las condiciones de equidad de los pueblos originarios. Sobre esta base, las medidas adoptadas para hacer conciencia 
de la diversidad cultural y garantizar algunos derechos elementales han sido impulsadas en muchas ocasiones por miembros destacados del gremio antropológico.

En este ámbito también hemos sido testigos de algunos avances en la política gubernamental en el campo del respeto a los derechos humanos. En la Comisión Nacional de Derechos Humanos (CNDH), un hasta hoy reducido grupo de antropólogos ha colaborado, como asesores o como trabajadores de este organismo. La CNDH, respondiendo a la delicada situación de los derechos humanos de los pueblos indígenas, creó en 1992 la Coordinación de Asuntos Indígenas para la atención de este segmento de la población mexicana. A partir de febrero de 1998 comenzó a operar como la Cuarta Visitaduría General, es decir un área especializada en la protección, la defensa, la promoción y la difusión de los derechos humanos de los pueblos indígenas del país. Lastimosamente, en esta instancia sólo trabaja una antropóloga que debe negociar, explicar y sensibilizar al grupo de abogados que allí labora y para quienes la problemática indígena es poco conocida.

Un rubro que se debe destacar es el referido a las modificaciones a la legislación relacionada con los códigos de procedimientos penales, tanto federal (1992) como local, que han abierto un campo de investigación y trabajo relacionado con la impartición de justicia en México. Entre los cambios realizados se señala que en los juicios en los que estén involucrados miembros de/o pueblos indígenas se debe contar con un traductor que permita al indiciado (inculpado) conocer los términos del juicio en su propio idioma. Así mismo, la nueva ley considera la posibilidad de allegar a los jueces dictámenes periciales que permitan emitir un juicio considerando los elementos culturales que pudieran servir como prueba en el proceso judicial. En este sentido, la presentación de peritajes antropológicos, culturales y lingüísticos ha brindado la oportunidad de que los "saberes antropológicos" ayuden a celebrar procesos jurídicos más justos. Este es uno de los campos profesionales más ricos que se está abriendo para todas las disciplinas antropológicas. Por ello para las escuelas y los colegios de antropología constituye uno de los retos más importantes que deben enfrentar a corto plazo. La difusión de este campo profesional, la capacitación y el reconocimiento de peritos, así como la oferta de una actualización constante de conocimientos son elementos centrales que deben tenerse en consideración para atender este 
campo. Al mismo tiempo, vamos a requerir de la adecuación de nuestras metodologías de trabajo tradicionales para incidir en la arena jurídica y vamos a tener que introducir una discusión seria sobre los dilemas éticos implicados en este tipo de actividad. Todos estos son elementos que aún no se incluyen adecuadamente en la formación profesional de los antropólogos ${ }^{15}$.

En lo que corresponde al ámbito de la procura de justicia en México, en la Procuraduría General de la República se creó la Unidad especializada para la atención de asuntos indígenas, mientras que en la Cámara de Diputados del Congreso de la Unión se estableció una Comisión de asuntos indígenas. Tenemos pues adelantos en materia de políticas públicas y en materia legislativa. Sin embargo, estos cambios son acotados y están lejos de resolver las demandas del movimiento indígena organizado. No obstante, lo más importante es que aún debemos resolver que estas modificaciones legislativas se lleven a la práctica y se definan los mecanismos específicos para darles cabal cumplimiento, para que los pueblos puedan ejercer sus derechos reconocidos ${ }^{16}$. Así mismo, es imprescindible reducir los niveles de pobreza e inequidad que reinan en México, particularmente en el medio rural, fortalecer

I5. El trabajo de peritaje antropológico se ha enriquecido enormemente de las experiencias existentes en Colombia, que pareciera ser el país latinoamericano que cuenta con mayor experiencia. La antropóloga colombiana Esther Sánchez Botero ha participado en diversas ocasiones en congresos y seminarios en México, compartiendo sus experiencias sobre este aspecto (véase Sánchez Botero, 200I).

I6. Para un recuento sobre los avances, los retos y las limitaciones de las políticas multiculturales desarrolladas en México, véase Valladares (2007). una cultura de respeto por los derechos humanos de los pueblos indígenas y una conciencia de la importancia de la diversidad cultural. En todas estas tareas resulta imprescindible contar con la visión antropológica, como ha comenzado a reconocerse. De ahí que en la actualidad los estudios específicos se realizan tanto en el sector gubernamental, como en las organizaciones sin ánimo de lucro e inclusive en empresas privadas de consultoría y asesoría. Pero como podemos ver, el proceso de reconocimiento de todas estas instancias sobre la necesidad de contar con estudios específicos con la visión antropológica es muy lento y heterogéneo.

Al difundirse las potencialidades del trabajo etnográfico y las visiones desde abajo, han surgido nuevos "clientes" para el trabajo antropológico. Estos pueden encontrarse hoy en empresas privadas que desarrollan estrategias de comercio justo o esquemas de proveedores en condiciones de equidad -como puede ser el caso 
de los productores de cultivos orgánicos, cooperativistas o grupos solidarios-. Pero también se sitúan en empresas totalmente alejadas de las preocupaciones de justicia social, equidad e incluso responsabilidad social, en la búsqueda de nuevos mercados y el desarrollo de productos para públicos muy específicos. Todos estos son nichos de empleo para el gremio, pero en todos debemos poner atención cuidadosa a la problemática ética de nuestro desempeño disciplinario. Aun cuando tradicionalmente hemos considerado que la forma en la que hacemos nuestro trabajo y la esencia misma de la disciplina (véase Krotz, 2002) pueden garantizar una visión general y un compromiso social amplio, esto no debe tenerse como un absoluto. Contamos con muchos ejemplos, tanto en México como en el ámbito internacional, de científicos sociales que transgreden las normas éticas elementales y la antropología no necesariamente es la excep-

I7. Véase el número 98 de la revista Relaciones de El Colegio de Michoacán, dedicado a la discusión de problemas éticos en antropología (http://www. colmich.edu.mx/relaciones/). ción ${ }^{17}$. El papel de los colegios profesionales es central en la definición de las normas éticas fundamentales y en la instrumentación de los mecanismos que permitan hacerlas respetar.

\section{Algunas experiencias concretas DE ANTROPÓLOGOS EN EL CAMPO PROFESIONAL}

HORA QUISIÉRAMOS REFERIRNOS MÁS PUNTUALMENTE AL VÍNCULO DE LA
antropología con la sociedad. Un número importante de colegas
está participando de manera activa, cercana -a veces median-
te el acompañamiento y otras con asesorías- a movimientos y
organizaciones sociales que luchan por instaurar una sociedad
más democrática en donde el respeto por la pluralidad sea una
realidad y no mera retórica. Uno de los campos más fértiles en
este rubro son las diferentes organizaciones no gubernamentales
u organismos de la sociedad civil. Estas organizaciones, que tie-
nen entre sus activistas a diversos antropólogos, se dedican a la
difusión, la promoción y la defensa de los derechos humanos y
los derechos civiles en general. En este ámbito, nuestros colegas
impulsan sendos programas de capacitación y asesoría para la
construcción de proyectos en salud, educación, organización de 
los productores rurales, los artesanos y también las empresas de comercio justo. En menor medida, el gremio cuenta con profesionales que trabajan en empresas de investigación de mercado y algunos también han abrazado la participación política activa en puestos de dirección o de elección popular.

En 2002, un grupo de estudiantes de la Escuela Nacional de Antropología e Historia hizo una serie de entrevistas a diez profesionales de la antropología dedicados a actividades no académicas. Con base en este material, Ma. de Lourdes Fernández Serratos y David Octavio Galavíz Magallanes redactaron un ensayo que fue publicado en el Boletín 6 del Ceas. El énfasis está puesto en las experiencias laborales de los entrevistados y en su perspectiva sobre la utilidad de su formación básica para hacer frente a necesidades laborales diversas. Los entrevistados se desempeñaban en puestos de nivel medio y alto e incluían un funcionario de una delegación política -equivalente al gobierno municipal dentro de la ciudad de México-, una comisionada de la Comisión Nacional de Derechos Humanos, un coordinador académico de una universidad, una consultora del Banco Mundial, una subdirectora y una coordinadora de investigación de un centro de investigación, una coordinadora del Consejo de Cronistas de una delegación política, una jefa de asesores de la oficina presidencial encargada de atención a la población indígena, un funcionario del Instituto Nacional de la Juventud, una investigadora de tiempo completo en una universidad y consultora.

El grupo no es muy amplio e incluye un buen número de antropólogos en tareas de administración académica y gestión pública. Sin embargo, las líneas de exposición del ensayo son muy interesantes porque resaltan, en palabras de los propios entrevistados, la importancia del antropólogo en tareas que requieren recuperar la voz y la visión de los actores. La mayor parte de los entrevistados consideraron que la formación del antropólogo permitía justamente acercarse al otro, rescatar su voz, respetar su visión y hacérsela comprender a terceras personas e instancias. Esto puede lograrse gracias a los métodos de la antropología basados en la observación, la participación y el análisis del contexto social e histórico de las personas y grupos con los que se trabaja, así como con base en la perspectiva holística de la disciplina. Al mismo tiempo, como señalamos antes, la cercanía con la población y nuestra mirada nos hace sensibles a las relaciones de poder en la vida cotidiana. 
Nos hace necesariamente críticos y señaladamente molestos para el statu quo.

Al mismo tiempo, hay herramientas que no necesariamente están hoy en el curriculum de la antropología y que resultan fundamentales cuando se trabaja fuera del ámbito académico. Uno de los puntos centrales es la administración del tiempo, la organización y periodización adecuada de nuestras actividades, el acotamiento preciso del problema que se analiza y el cumplimiento de límites precisos y reducidos de tiempos de entrega de informes o resultados.

Por otra parte, debe subrayarse que el hecho de que el principal empleador de antropólogos fuera, durante mucho tiempo el estado mexicano, tanto en el terreno académico como en el profesional, ha resultado en la reducción de nuestro campo de trabajo a medida que el estado se contrae y traslada algunas de sus funciones a la iniciativa privada. Los antropólogos entrevistados señalan que cada vez es más necesario revalorar la disciplina para promover la inserción de antropólogos en espacios de trabajo no tradicionales. Es necesario promover nuestra disciplina y en ocasiones generar nuestras propias fuentes de empleo, como es el caso de los consultores independientes. En este rubro, de nuevo, aparecen algunas carencias en la formación tradicional que es importante revisar y subsanar. Fernández Serratos y Galavíz Magallanes subrayan la necesidad de que los antropólogos obtengan de su formación básica herramientas para lograr mayor precisión en el análisis y la delimitación de problemas concretos, aptitud para el trabajo multidisciplinario, el empleo de herramientas cuantitativas, instrumentos para la comprensión, el análisis y la conceptualización de las relaciones entre lo local y lo global; la capacidad para interrelacionar lo micro y lo macro; el empleo de herramientas tecnológicas digitales para la generación, la organización, el análisis y la presentación de datos; y competencia en la lectura, la redacción y la comunicación oral en varios idiomas. El hecho de que muchas de estas habilidades no se enseñen hoy a los antropólogos provoca que tengan menos oportunidades en el mercado laboral y los obliga a obtener una formación complementaria cuando se insertan en el terreno profesional. 


\author{
El Colegio de Etnólogos \\ y Antropólogos Sociales, AC
}

N MÉXICO, LOS COLEGIOS PROFESIONALES -O COLEGIOS DE PROFESIONISTAS,
Como también se les llama- son asociaciones civiles -no lucra-
tivas-formadas por egresados de una misma rama disciplinaria interesados en agruparse para trabajar en beneficio de su rama profesional y del mercado de trabajo que les corresponde atender. $\mathrm{Al}$ hacerlo y ser reconocidos mediante un registro federal que otorga la Secretaría de Educación Pública, son responsables de promover acciones en beneficio de la población, esencialmente mediante el servicio social profesional, desempeñando tareas directamente relacionadas con su quehacer, con el propósito de elevar la calidad de vida de la comunidad. Deben vigilar también el ejercicio profesional con el objeto de que éste se realice dentro del más alto plano legal y ético. Para lograrlo están facultados para promover la expedición de leyes, reglamentos -y sus reformas- relativos al ejercicio profesional así como colaborar con las instituciones de enseñanza superior en la elaboración de planes de estudios adecuados y pertinentes a las transformaciones que requiere el país y sus tareas profesionales.

En esta última competencia los colegios de profesionistas ligados a la antropología que existen en México -el Colegio de Etnólogos y Antropólogos Sociales AC (Ceas); la Sociedad Mexicana de Antropología (SMA); el Colegio Mexicano de Antropólogos (CMA); la Asociación Mexicana de Antropología Biológica AC, entre otros- deben participar de manera activa y comprometida con los retos que hoy tiene la antropología frente a la formación de nuevos antropólogos, en la que se mantenga una sólida formación teóricametodológica, así como una continua actualización y preparación para las nuevas exigencias del mercado profesional. No menos importante es fortalecer el papel de los colegios como portavoces del gremio nacional y recuperar la función de interlocutores con el aparato gubernamental que tuvieron en décadas pasadas.

El Ceas, constituido desde 1976, asume estos compromisos para los campos de la etnología, la antropología social y la etnohistoria. Considera así entre sus objetivos centrales vigilar y procurar activamente el ejercicio de la profesión; promover y divulgar la investigación básica y aplicada en estas tres subdisciplinas; propugnar porque sus socios obtengan retribuciones justas por su 
trabajo profesional; servir de árbitro en casos de conflicto entre profesionales de las disciplina; y fomentar las relaciones con otros colegios de profesionistas nacionales y extranjeros.

Para el logro de estos objetivos, el Ceas promueve actividades de formación, capacitación profesional y divulgación de las actividades de la disciplina. Durante el último bienio ha realizado un gran avance en términos de su consolidación como asociación profesional. Sus órganos de difusión, las publicaciones y la organización misma se han visto fortalecidas. Entre sus actividades regulares debe destacarse la publicación del Boletín, que se ha vuelto una publicación regular esperada con interés por todos los miembros, por sus artículos analíticos sobre alguno de los campos profesionales. Asimismo, el boletín electrónico VOCEAS ha tenido un papel central en la circulación de noticias e información relacionada con la antropología en México y el mundo; la serie de Biografías de los maestros de la antropología mexicana publicada durante el último decenio ha informado a las nuevas generaciones sobre los aportes y logros de un grupo de personajes cuya trayectoria fue fundamental para constituir a la disciplina en el siglo veinte; por último, la participación de sus socios en la organización de encuentros y reuniones temáticas relacionadas con su quehacer ha permitido dialogar con instituciones, sectores sociales y con otros profesionistas sobre las tareas por emprender y los enfoques más adecuados para ello.

En el futuro el Ceas debe mantener y fortalecer su presencia en el gremio antropológico, tanto en lo que se refiere a la promoción de la disciplina como en el esfuerzo por hacer visible el amplio abanico de tareas profesionales que desempeñan los antropólogos. Es importante que impulse la reflexión sobre el papel que debe desempeñar la antropología en el mundo del siglo veintiuno, un mundo marcado por la globalización, la multipolaridad y la multiculturalidad poscolonial. Debe, así mismo, continuar impulsando el reconocimiento de las respuestas que la antropología propone diariamente para los problemas del mundo contemporáneo en sus diferentes ámbitos de acción. El objetivo primordial de estas tareas es coadyuvar al fortalecimiento de la actividad profesional de la antropología mediante una serie de acciones centradas en la promoción de la disciplina, la difusión del conocimiento generado y en actividades tendientes a la actualización profesional y la discusión de los diversos y complejos retos que enfrenta. 
Entre estos últimos podemos destacar la discusión y puesta en práctica de una serie de principios éticos para los profesionales de la disciplina, una mayor vinculación con las instituciones de investigación y formación de antropólogos. Del mismo modo, es fundamental hacer más abundantes y sólidos los vínculos con diferentes colegios de profesionistas y asociaciones profesionales de antropólogos, tanto de México como del extranjero. Sólo con estos lazos será posible participar en los procesos de evaluación, certificación y actualización de las actividades antropológicas, entre ellas las vinculadas con el peritaje antropológico.

Así mismo, es fundamental mantener actualizados los registros de los socios del Ceas, con el objeto de monitorear los distintos campos profesionales y los nichos de mercado en los que se insertan, ya sean tradicionales -diversas dependencias del sector público-o novedosos -sectores sociales y privados-, con el objeto de incorporar sus experiencias, apreciaciones, condiciones y retos que enfrentan como antropólogos y lograr su mejor desenvolvimiento y una mayor presencia en las discusiones de los grandes asuntos nacionales.

\section{Conclusiones}

L MUNDO ES DIVERSO Y LAS RAZONES PARA QUE UN ANTROPÓLOGO SE HAYA insertado en algún nicho laboral de los arriba mencionados van desde la casualidad, la oportunidad y las redes personales, hasta la verdadera búsqueda de las capacidades analíticas y metodológicas de la formación antropológica. En no pocos casos puede subrayarse, como hicieron los entrevistados que mencionamos arriba, que los antropólogos han contribuido a crear nuevas fuentes de trabajo en campos no tradicionalmente atendidos por la antropología.

Con este breve panorama del campo laboral profesional en el México contemporáneo pretendemos ahondar en lo que hace décadas atinadamente señalaba Palerm: no podemos hacer una distinción contundente entre antropólogos teóricos y antropólogos aplicados. Si reconocemos inicialmente que en gran medida los estudios antropológicos tienen que ver con la alteridad sociocultural; si nuestro trabajo consiste en estudiar todos los fenómenos sociales desde la perspectiva de la alteridad, es muy probable que desarrollemos una ciencia social con un carácter 
esencialmente crítico y hasta subversivo. Este carácter no radica, como nos recuerda Krotz, en la estructura de personalidad o en la oposición política o social de este o aquel de sus practicantes o de sus organizaciones gremiales (Krotz, 2002: 28-29). Es probable que sean la esencia y el propósito mismos de la disciplina lo que nos conduce en esta dirección, en tanto que nuestra ciencia estudia cualquier rasgo cultural, acción social, institución o proceso histórico como algo que en otra parte o en otro tiempo podría existir -y de hecho, casi siempre se puede demostrar que existe- de modo diferente. Por ello inclusive la simple etnografía descriptiva constituye un reto para la sociedad en la que se difunde, porque despoja sus instituciones y su cosmovisión del halo de naturalidad e inmutabilidad en que se basan normalmente los procesos de enculturación y legitimación y que constituye siempre también un mecanismo de inmunización de cualquier sociedad ante los impulsos de cambio. El conocimiento antropológico pone en crisis a la sociedad, pues exige que sea justificado explícitamente el orden vigente, que en mayor o en menor medida es desenmascarado como posible de otra forma (Krotz, 2002: 29). De suerte tal que desde la academia y desde otras trincheras profesionales la antropología continúa siendo una ciencia crítica y propositiva.

En esta discusión resulta central subrayar que la especificidad de la antropología se basa en la combinación de dos elementos centrales: la yuxtaposición de formas diferentes de ser, de entender el mundo y actuar sobre él; y un entrenamiento profesional que parte de la valoración de estas diferencias y subraya la importancia de su existencia para la vida humana y la continuidad de la existencia de las sociedades humanas. Estos elementos están en el origen de una serie de herramientas metodológicas que son consustanciales a una forma de práctica tanto en la investigación como en la aplicación de los conocimientos adquiridos. Los métodos de trabajo de la disciplina, por sí mismos, no garantizan que la antropología sea una disciplina científica crítica y propositiva. La práctica misma no garantiza tampoco la rectitud de sus agentes profesionales ni la observancia de normas éticas en su comportamiento. La diversificación del campo laboral debe llevarnos entonces a atender estos puntos: una formación teórica y metodológica sólida, experiencia vasta y supervisada en el trabajo de campo, además de herramientas novedosas especializadas que permitan atender necesidades que van de la medicina al derecho, de la economía a la política, de la educación a las actividades artísticas. 
En el inicio del nuevo milenio se nos plantean problemas sociales que representan retos fundamentales para la disciplina: migración, inequidad y pobreza estructurales, multiculturalidad y cosmopolitismo, intolerancia, incomprensión y fanatismo armado, por mencionar sólo algunos. Como señalamos arriba, los colegios profesionales en México tienen entre sus funciones primordiales trabajar en beneficio de su rama profesional y del mercado de trabajo que les corresponde atender. Con base en los principios del Ceas y con la mirada puesta en el futuro, nos hemos propuesto dar continuidad a sus actividades y establecer algunos mecanismos que permitan mantener su dinamismo. El objetivo primordial es coadyuvar al fortalecimiento de la actividad profesional de la antropología mediante una serie de acciones centradas en la promoción de la disciplina, la difusión del conocimiento generado y en actividades tendientes a la actualización profesional y la discusión de los retos que enfrenta. En suma, debemos refrendar el papel central que la antropología debe, y puede, desempeñar en la mejor comprensión y en el planteamiento de soluciones a los problemas de nuestro tiempo.

En esta misma época, los cambios de la antropología reflejan una diversidad creciente de interpretaciones, de elaboraciones teóricas y de propuestas de soluciones prácticas a los problemas del mundo. Frente a estos retos del presente, como antropólogos debemos ser concientes de que somos una colectividad significativa, que se requieren nuevos espacios laborales y que debemos velar porque las universidades y otras instituciones formadoras mantengan una sólida formación teórica y metodológica para los estudiantes. Esto implica nuestra participación en la diversificación del mercado laboral y también en la revisión y actualización de los currículos, para responder a los retos interpretativos y prácticos de una sociedad cada vez más diversa social y políticamente. Si la antropología ha de contribuir con soluciones apropiadas a los problemas contemporáneos, a la formación de sociedades multiculturales y democráticas, debe aportar herramientas y profesionales para un mercado de trabajo diversificado y complejo. Es necesario participar en la construcción, el análisis y la evaluación de las posibles contribuciones de la antropología a los nuevos campos del conocimiento y de la praxis, pero es igualmente urgente identificar los retos y las implicaciones de esta práctica en términos de los derechos y deberes y la ética. 


\section{Fuentes citadas}

Aguirre Beltrán, Gonzalo. "De eso que llaman antropología mexicana”. En Obra polémica. México: CISINAH, I975.

Anuies. Anuario estadístico. http://www.anuies.mx/servicios/e_ educacion/docs/anuario_estadistico_2004_licenciatura.pdf, 2004.

ArizPe, Lourdes, coordinadora. Antropología breve de México. México: Academia de la Investigación Científica-Crim-Unam, i993.

BÁEz Landa, Mariano. "La ciencia imposible. La antropología mexicana entre la ciencia y la acción”. Ponencia presentada en La otra antropología toma la palabra, UAM-Iztapalapa, 2I y 22 de septiembre 2005. Documento del Seminario miradas antropológicas, visitado el Io de enero de 2007, http://www.ciesas-golfo.edu.mx/seminarios/ mbaez/miradas/_Media/ciencia_imposible.pdf).

Bensusán, Graciela e Ívico Ahumada Lobo. “Sistemas de jubilación en las instituciones públicas de educación superior y composición por edad del personal académico”. Revista de la Educación Superior, XXXV (2) I38 (2006): 7-33.

Bonfil, Guillermo et al. De eso que llaman antropología mexicana. México: Editorial Nuestro Tiempo, I970.

Colegio de Etnólogos y Antropólogos Sociales A.C. Página de internet. http://www.ceas.org.mx/

Consejo Mundial de Asociaciones de Antropología (WACC). http://www. wcaanet.org/

Del Val, José. México. Identidad y nación. México: Unam, 2004.

Díaz-Polanco, Héctor. Elogio de la diversidad. Globalización, multiculturalismo y etnofagia. México: Editorial Siglo XXI, 2006.

Dietz, Gunther. Multiculturalismo, interculturalidad y educación: una aproximación antropológica. México: Ciesas-Universidad de Granada, 2003

Escamilla Hurtado, Ma. Guadalupe. "La formación contemporánea de antropólogos sociales y etnólogos en México. Inventario de escuelas y facultades". Boletín del Colegio de Etnólogos y Antropólogos Sociales A.C., 2 (I999): 9-I8.

Fernández Serratos, Ma. de Lourdes y David Octavio Galavíz Magallanes. "Formación y retos del campo laboral: una reflexión". Boletín del CEAS, (6) (2002): IO-I8.

FREYERMUTH EnCISO, GRACIELA. "La mortalidad maternal y los nudos en la prestación de los servicios de salud. Un análisis desde la interculturalidad". s. f. http://www.ciesas.edu.mx/lerin/doc-pdf/Freyermuh-II.pdf 
Gardner, K. y D. Lewis. Anthropology, development and the post-modern challenge. Londres: Pluto Press, I996.

Gros, Chistian. Políticas de la etnicidad: identidad, estado y modernidad. Bogotá: Instituto Colombiano de Antropología e Historia, 2000.

Hernández Castillo, AídA. “¿Conocimiento para qué? La antropología crítica: entre las resistencias locales y los poderes globales. Discurso de recepción de la "Martin Diskin Lectureship at LASA 2003"”. LASA Forum, 34 (3) (2003): 5-9.

Hernández Castillo, Aída, Sarela Paz y Teresa Sierra, coordinadoras. El Estado y los indígenas en tiempos del PAN: neoindigenismo, legalidad e identidad. México: Editorial Cámara de Diputados-Porrúa-Ciesas, 2004.

Hewitt, Cynthia. Imágenes del campo. La interpretación antropológica del México rural. México: El Colegio de México, I984.

Krotz, Esteban. "Sociedades, conflictos, cultura y derecho desde una perspectiva antropológica”. En Antropología jurídica: perspectivas socioculturales en el estudio del derecho, editado por Esteban Krotz. Barcelona: Anthropos-Universidad Autónoma MetropolitanaIztapalapa, 2002.

"Historia e historiografía de las ciencias antropológicas: una problemática teórica”. En La antropología en México. México: Inah. Tomo I, I987.

LAmeiras, José. "La antropología en México. Panorama de su desarrollo en lo que va del siglo”. En El Colegio de México, editor. Ciencias sociales en México. Desarrollo y perspectiva. México: El Colegio de México, I979.

Lerín Piñón, SERgio. "Desafíos de la salud intercultural”. http://www. geocities.com/congresoprograma/8-I.pdf, s. f.

López BÁRCENAS, Francisco. Legislación y derechos indígenas en México. México: Ceacatl-Red-es-Ediciones Casa Vieja-Centro de Orientación y Asesoría a Pueblos Indígenas AC. Serie Derechos Indígenas 3, 2002.

Medina, Andrés y CARlos García Mora, coordinadores. La quiebra política de la antropología social mexicana. México: Unam. I983.

Molina, Virginia. "La antropología aplicada en la formación de antropólogos”. Documento del seminario Miradas antropológicas, visitado el to de enero de 2007, http://www.ciesas-golfo.edu.mx/ seminarios/mbaez/miradas/_Media/vmolina.pdf, s. f.

Nolasco, Margarita. Los sujetos de estudio de la antropología. México: Ediciones de la Casa Chata-Ciesas. Número Io9. 
OeHmichen, Cristina. Reforma del Estado, política social e indigenismo en México (I988-1996). México: Instituto de Investigaciones Antropológicas, Unam, I999.

PALERM, ÁngEL. "Antropología aplicada y desarrollo de la comunidad”. En Planificación regional y reforma agraria, Á. Palerm. México: Universidad Iberoamericana-Ediciones Gernika, I993.

“La disputa de los antropólogos mexicanos: una continuación científica”. América Indígena, XXXV (I) (I985): I6I-I77.

REBOlLEDO, NicAnor. "Autonomía indígena y educación intercultural. Anuario Educativo Mexicano: una visión retrospectiva”. Universidad Pedagógica Nacional (UPN), México. http://interbilingue.ajusco.upn. $\mathrm{mx} /$ modules.php?name $=$ News\&file $=$ article\&sid $=83,200 I$.

Salmerón Castro, Fernando I. "Un rol necesario en un momento oportuno. Entrevista a Gonzalo Aguirre Beltrán”. En Caminos de la antropología. Entrevistas a cinco antropólogos, compilado por J. Durand y Luis Vázquez. México: Dirección General de Publicaciones del Consejo Nacional para la Cultura y las Artes-Instituto Nacional Indigenista, I990.

SÁnchez, Consuelo. Del indigenismo a la autonomía. México: Siglo XXI, I999.

SÁnchez Botero, Esther e IsAbel Cristina Jaramillo Sierra. La jurisdicción especial indígena. Bogotá: Procuraduría General de la Nación, Procuraduría delegada para minorías étnicas, $200 I$.

Schmelkes, Sylvia. "La educación superior intercultural: el caso de México”. http://www.anuies.mx/e_proyectos/pdf/La_educ_sup_ indigena. pdf, 2003.

Secretaría del Trabajo y Previsión Social (STPS). Página electrónica del Observatorio Laboral http://www.observatoriolaboral.gob.mx/ PanoramaCar.asp?clave $=253$

SEP-CGEIB. Universidad intercultural. Modelo educativo. México: Secretaría de Educación Pública-Coordinación General de Educación Intercultural y Bilingüe, 2006.

-Educación superior para los pueblos indígenas de América Latina. Memorias del segundo encuentro regional. México: Secretaría de Educación Pública-Coordinación General de Educación Intercultural y Bilingüe-Unesco, 2004.

VALLADARES, LAURA. "El movimiento indígena en México: constructores y excluidos de la democracia”. En Política, etnicidad e inclusión digital en los albores del milenio, editado por Héctor Tejera, Scott Robinson y Laura Valladares. México: Editorial Porrúa-UAM, 2007. 
"Democracia y derechos indios en México: la ciudadanía multicultural como modelo de paz". Revista Mexicana de Ciencias Políticas y Sociales. 2003: I2I-I46.

VÁZquEZ LEÓN, LuIs. "La historiografía antropológica contemporánea en México”. En La antropología en México. México: Inah. Tomo I, I987. 